\title{
Simulación de la interacción entre fracturas hidráulicas y fracturas naturales con aplicación de daño continuo
}

\section{Simulation of the interaction between hydraulic fractures and natural fractures with application of continuum damage}

\author{
Anny Zambrano-Luna ${ }^{1}$, Germán González-Silva ${ }^{2}$, Yair Quintero-Peña ${ }^{3}$ \\ ${ }^{1}$ Grupo de investigación de Estabilidad de Pozos, Escuela de Ingeniería de Petróleos, Universidad Industrial de Santander, \\ Colombia. Email: anny.zambrano@correo.uis.edu.co \\ ${ }^{2}$ Grupo de Modelamiento de Hidrocarburos, Escuela de Ingeniería de Petróleos, Universidad Industrial de Santander, Colombia. \\ Email: germangs@uis.edu.co \\ ${ }^{3}$ 1 Grupo de investigación de Estabilidad de Pozos, Escuela de Ingeniería de Petróleos. Instituto Colombiano del Petróleo, \\ Piedecuesta, Colombia. Email: yair.quintero@ecopetrol.com.co
}

ReCiBIDO: Marzo 17, 2017. ACEPTADO: Agosto 13, 2017. Versión Final: Septiembre 27, 2017.

\begin{abstract}
RESUMEN
El concepto de daño continuo es usado para estudiar la interacción entre fracturas hidráulicas y fracturas naturales a través del planteamiento de un modelo, cuyo objetivo es representar el camino de propagación y la relación entre estos dos tipos de fracturas, así como, predecir su complejo comportamiento sin la necesidad de predefinir su dirección como ocurre en otras aplicaciones de elementos finitos, proporcionando resultados más consistentes con el comportamiento físico del fenómeno. El enfoque usa simulaciones de elementos finitos a través del software Abaqus para modelar el fracturamiento por daño o el proceso de fracturamiento por propagación de daño en una roca. El modelado del fenómeno se desarrolla en dos dimensiones (2D) de manera que la fractura será representada por una línea y el frente de fractura por un punto. Se considera comportamiento constitutivo no-lineal, deformación finita, deformación dependiente del tiempo, deformación hardening y softening, y deformación basada en la evolución del daño en compresión y tensión.
\end{abstract}

PALABRAS CLAVE: Red de fractura compleja; daño continuo; fracturas hidráulicas; fracturas naturales; rigidez.

\begin{abstract}
The continuum damage concept is used to study the interaction between hydraulic fractures and natural fractures through a model, whose objective is to represent the path of propagation and the relationship between these two types of fractures, as well as predict their complex behavior without the need to predefine its direction as it occurs in other applications of finite elements, providing results more consistent with the physical behavior of the phenomenon. The approach uses finite element simulations through Abaqus software to model damage fracturing or fracturing process by damage propagation in a rock. The modeling the phenomenon develops in two dimensional (2D) so that the fracture will be represented by a line and the crack front by a point. It considers nonlinear constitutive behavior, finite strain, time-dependent deformation, strain hardening and softening, and strain based damage evolution in compression and tension.
\end{abstract}


KEYWORDS: Complex fracture network; continuum damage; hydraulic fractures; natural fractures; stiffness.

\section{INTRODUCCIÓN}

La interacción entre una fractura hidráulica y una fractura natural preexistente, parece ser el componente clave que explique por qué algunos yacimientos exhiben un comportamiento más complejo [1]. El diseño de fracturas hidráulicas convencionales está basado en la suposición de que la roca es homogénea y la fractura se propaga simétricamente en un plano perpendicular al esfuerzo mínimo, en yacimientos naturalmente fracturados debido a la interacción con fracturas naturales, pueden propagarse asimétricamente o en múltiples ramas o segmentos, generando una red de fracturas complejas en el sistema [2]. Incluso, el fracturamiento hidráulico de una formación que puede ser idealizada como homogénea, isotrópica y continua involucra procesos complejos, no-lineales e hidromecánicos a diferentes escalas [3].

Los yacimientos de shale gas y shale oil en las escalas de tiempo y longitud de interés durante estimulaciones con fracturamiento hidráulico, no pueden ser aproximadas como homogéneas, debido a la crítica afectación resultado de la interacción entre las fracturas hidráulicas $(\mathrm{FH})$ y las fracturas naturales (FN) presentes en el medio. Esta interacción afecta no solo la velocidad de la propagación de la $\mathrm{FH}$, sino también la estimulación del yacimiento caracterizado, donde las FN sufren deformación inelástica (i.e. deslizamiento y apertura) [3]. De manera que, para analizar y diseñar un tratamiento de fracturamiento hidráulico es necesario tener herramientas capaces de simular la propagación de las fracturas en masas rocosas discontinuas (ya fracturadas).

El modelo utilizado consiste en la implementación del método de elementos finitos (MEF) con mecánica de daño continuo, en el cual la fractura es representada por elementos continuos cuya resistencia es reducida a un mínimo valor y la permeabilidad de los elementos fisurados puede ser relacionada a la deformación o al estado de esfuerzos correspondiente [4]; este método es capaz de simular efectos no planares o fuera de plano [5] lo que permite observar la geometría compleja de fractura generada y la propagación de una FH bajo condiciones geomecánicas complejas, facilitando un verdadero análisis para el fenómeno en estudio. Para describir la geometría de FH-FN final a través de un modelo de simulación, se presenta la formulación de daño y el modelo constitutivo, el cual incorpora un criterio de cedencia dependiente de la presión, una regla de flujo plástico, una regla hardening y daño. El enfoque elimina la dirección de propagación predefinida de la $\mathrm{FH}$, característica que limita el desarrollo real de la fractura y está presente en la mayoría de los trabajos que involucran elementos finitos en la materia; permitiendo la evaluación de la geometría de red compleja final, usando un software comercialmente disponible (Abaqus). La simulación fue comparada con trabajos publicados en la literatura.

\section{DAÑO Y DEGRADACIÓN DE LA RIGIDEZ}

El modelo de daño [6], [7] incluido en Abaqus [8], [9] aplica el concepto de módulo de deformación (E) y reducción de rigidez, usando el parámetro de daño, $d$, como una aproximación adimensional de la degradación de la rigidez para escalar el verdadero esfuerzo. En la etapa inicial, $D=D_{0}, d=0$ (sin degradación); y en falla, $d=1$, el material es completamente dañado y el esfuerzo efectivo disminuye a cero [10].

$$
E^{\prime} / E_{0} \approx D^{\prime} / D_{0}=(1-d)
$$

La deformación plástica incremental incluye todas las deformaciones irreversibles, así como el daño por microfisuramiento frágil. La descomposición de la deformación en los componentes elástico y plástico $(\varepsilon=$ $\varepsilon^{e}+\varepsilon^{p}$ ) da la relación esfuerzo-deformación:

$$
\sigma=(1-d) D_{0}\left(\varepsilon-\varepsilon^{p}\right)
$$

El concepto de esfuerzo efectivo en está ocasión (no está relacionado con la presión de poro) es usado para degradar la rigidez elástica, que a su vez controla la forma de la superficie de cedencia (yield surface). El parámetro daño, evoluciona separadamente como una función de la deformación plástica y realiza un seguimiento por separado para el daño por tensión y compresión (Figura 1). El resultado es un material que conserva la resistencia direccional dependiendo de cómo sea forzado.

El modelo utilizado se denomina concrete damaged plasticity y proporciona una capacidad general para modelar concreto y otros materiales cuasi-frágiles en todos los tipos de estructuras, utilizando los conceptos de elasticidad y daño isotrópico combinados con plasticidad en tensión y compresión isotrópica para representar el comportamiento inelástico del material [9]. 


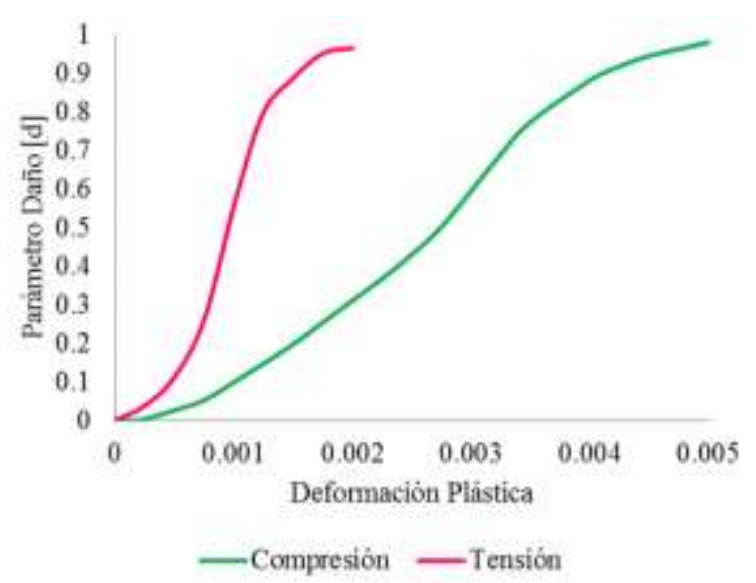

Figura 1. Daño progresivo. Fuente. [10].

Asume que las curvas de esfuerzo-deformación uniaxial pueden ser convertidas en curvas de esfuerzo versus deformación plástica de la forma:

$$
\begin{aligned}
& \sigma_{t}=\sigma_{t}\left(\tilde{\varepsilon}_{t}^{p l}, \dot{\tilde{\varepsilon}}_{t}^{p l}, \theta, f_{i}\right) \\
& \sigma_{c}=\sigma_{c}\left(\tilde{\varepsilon}_{c}^{p l}, \dot{\tilde{\varepsilon}}_{c}^{p l}, \theta, f_{i}\right)
\end{aligned}
$$

Donde los subíndices t y c hacen referencia a tensión y compresión, respectivamente. $\dot{\tilde{\varepsilon}}_{t}^{p l}$ y $\dot{\tilde{\varepsilon}}_{c}^{p l}$ son las tasas de deformación plástica equivalente, $\tilde{\varepsilon}_{t}^{p l}=\int_{0}^{t} \dot{\tilde{\varepsilon}}_{t}^{p l} d t \mathrm{y}$ $\tilde{\varepsilon}_{c}^{p l}=\int_{0}^{t} \dot{\tilde{\varepsilon}}_{c}^{p l} d t$ son las deformaciones plásticas equivalentes, $\theta$ es la temperatura y $f_{i},(i=1,2, \ldots)$ son otras variables de campo predefinidas. Se adopta la convención de que $\sigma_{c}$ es una cantidad positiva que representa la magnitud del esfuerzo de compresión uniaxial; es decir, $\sigma_{c}=-\sigma_{11}$.

Bajo condiciones de carga uniaxial las tasas de deformación plástica efectiva están dadas por:

$$
\begin{gathered}
\dot{\tilde{\varepsilon}}_{t}^{p l}=\dot{\varepsilon}_{11}^{p l} \\
\dot{\tilde{\varepsilon}}_{c}^{p l}=-\dot{\varepsilon}_{11}^{p l}
\end{gathered}
$$

Como se muestra en la Figura 2, cuando el material es descargado desde cualquier punto de la sección strain softening de la curva esfuerzo-deformación, se observa que la respuesta a la descarga es el debilitamiento: la rigidez elástica del material parece estar dañada o degradada. La degradación de la rigidez elástica es significativamente diferente entre las pruebas de tensión y compresión; en cualquier caso, el efecto es más pronunciado a medida que la deformación plástica aumenta. La respuesta a la degradación del material es caracterizada por dos variables de daño uniaxial independientes, $d_{t} \mathrm{y} d_{c}$, que se supone son funciones de la de las deformaciones plásticas, la temperatura y las variables de campo:

$$
\begin{aligned}
& d_{t}=d_{t}\left(\tilde{\varepsilon}_{t}^{p l}, \theta, f_{i}\right),\left(0 \leq d_{t} \leq 1\right) \\
& d_{c}=d_{c}\left(\tilde{\varepsilon}_{c}^{p l}, \theta, f_{i}\right),\left(0 \leq d_{c} \leq 1\right)
\end{aligned}
$$

Las variables de degradación uniaxial son funciones crecientes de las deformaciones plásticas equivalentes y, pueden tomar valores que van desde cero, para material no dañado, a uno, para material totalmente dañado. Si $E_{0}$ es la rigidez elástica inicial (no dañada) del material, las relaciones esfuerzo-deformación bajo cargas de tensión y compresión uniaxial son, respectivamente:

$$
\begin{aligned}
& \sigma_{t}=\left(1-d_{t}\right) E_{0}\left(\varepsilon_{t}-\tilde{\varepsilon}_{t}^{p l}\right) \\
& \sigma_{c}=\left(1-d_{c}\right) E_{0}\left(\varepsilon_{c}-\tilde{\varepsilon}_{c}^{p l}\right)
\end{aligned}
$$

Adicionalmente, los esfuerzos de cohesión uniaxial efectivos, $\bar{\sigma}_{t}$ y $\bar{\sigma}_{c}$, determinan el tamaño de la superficie de cedencia (o falla):

$$
\begin{aligned}
& \bar{\sigma}_{t}=\frac{\sigma_{t}}{\left(1-d_{t}\right)}=E_{0}\left(\varepsilon_{t}-\tilde{\varepsilon}_{t}^{p l}\right) \\
& \bar{\sigma}_{c}=\frac{\sigma_{c}}{\left(1-d_{c}\right)}=E_{0}\left(\varepsilon_{c}-\tilde{\varepsilon}_{c}^{p l}\right)
\end{aligned}
$$
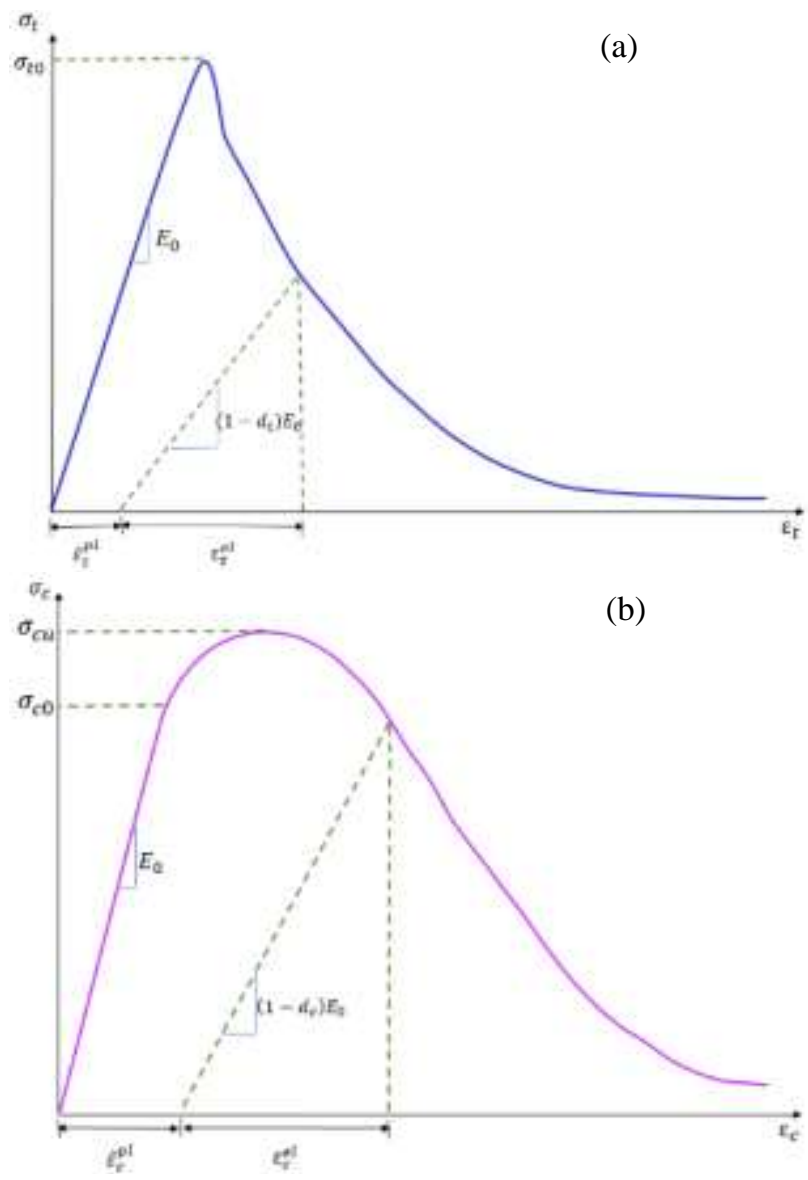

Figura 2. Respuesta del concreto a carga uniaxial en tensión (a) y compresión (b). Fuente. [9]. 


\section{MODELO CONSTITUTIVO}

Incorpora un criterio de cedencia dependiente de la presión, una regla de flujo plástico, una regla de hardening y daño. La combinación de strain softening y daño plástico permite la simulación de debilitamiento local extremo, donde las fracturas o zonas fracturadas ocurren donde el material se degrada a resistencia cero. El modelo está basado en modificaciones realizadas a la plasticidad clásica de Mohr-Coulomb, como se describe a continuación [10].

El comienzo de cedencia (yielding) que ocurre en la transición dúctil-frágil en rocas, es típicamente dependiente de la presión [11], [12], [13] donde el criterio de Mohr Coulomb describe esta relación vinculando el esfuerzo de corte y normal a través de un plano mediante la función:

$$
\tau=\mu \sigma_{n}+c
$$

Donde $\tau$ es el esfuerzo de corte, $\sigma_{n}$ es el esfuerzo normal, $\mu=\tan \emptyset$ permite conocer el coeficiente de fricción y el ángulo de fricción $y, c$ es la resistencia al corte o cohesión.

El criterio de Mohr Coulomb (Figura 3) asume que la falla no depende del esfuerzo principal intermedio; sin embargo, Mogi [15], Reches y Dieterich [14] mostraron que esta suposición no es necesariamente válida en el caso general. Asumiendo, que la superficie de cedencia (yield Surface) es totalmente dependiente del esfuerzo principal intermedio, $\tau_{o c t}$ es constante para todas las rotaciones de $\theta$, como se indica en el criterio de Drucker Prager, donde la forma de la superficie de cedencia es un cono que se abre con el aumento del esfuerzo promedio.

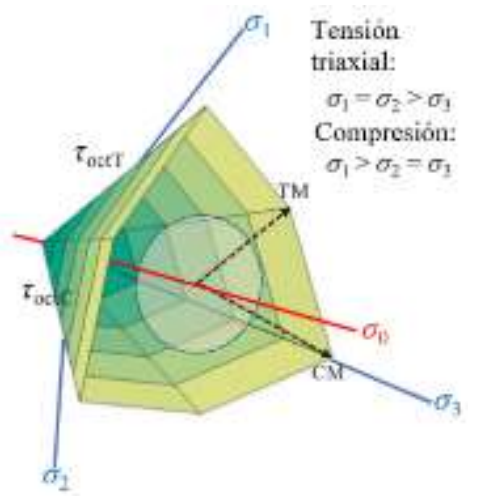

Figura 3. Superficie de cedencia de Mohr Coulomb que muestra los meridianos de tensión y compresión (TM y CM), definidos por el esfuerzo de corte octaédrico en tensión y compresión $\left(\sigma_{o c t T}\right.$ y $\left.\sigma_{o c t C}\right)$. Fuente. [10].
La superficie de falla de Drucker Prager es una variación dependiente de la presión en el criterio de Von Mises y está dada mediante:

$$
J_{2}^{1 / 2}=k+3 \alpha I_{1}
$$

Donde $\alpha$ y $k$ son constantes del material, y los esfuerzos invariantes en términos de los esfuerzos principales $\sigma_{1}$, $\sigma_{2}$ y $\sigma_{3}$ y el esfuerzo promedio $\sigma_{0}$ son:

$$
\begin{gathered}
I_{1}=\sigma_{1}+\sigma_{2}+\sigma_{3}=3 \sigma_{0} \\
J_{2}=3 \sigma_{0}-I_{2} \\
I_{2}=\sigma_{1} \sigma_{2}+\sigma_{2} \sigma_{3}+\sigma_{3} \sigma_{1}
\end{gathered}
$$

En el desarrollo del trabajo se utiliza la superficie de cedencia modificada de Lubliner [6], la cual combina las características positivas de los modelos de MohrCoulomb y Drucker Prager; llamada también, superficie de cedencia del modelo Barcelona (Figura 4) [16].

La superficie Drucker Prager (ecuación 14) ajusta dentro de la forma:

$$
F(\sigma)=1 /(1-\alpha)\left(\left[3 J_{2}\right]^{1 / 2}+\alpha I_{1}\right)
$$

Añadiendo dependencia en el esfuerzo más grande $\sigma_{\max }$ $\mathrm{y}$ asignando dos parámetros adicionales, $\beta<\sigma_{\max }>\mathrm{y}$ $\gamma\left\langle\sigma_{\max }>\right.$ a la ecuación 18, el círculo de Drucker Prager puede ser modificado reduciendo la dependencia del esfuerzo principal intermedio y estableciendo meridianos de tensión y compresión. Así, la forma presentada por Lubliner [6], Lee y Fenves [7] es:

$$
\begin{aligned}
& F(\sigma) \\
& =1 /(1-\alpha)\left(\left[3 J_{2}\right]^{1 / 2}+\alpha I_{1}+\beta<\sigma_{\max }>-\gamma<-\sigma_{\max }>\right)
\end{aligned}
$$

Donde [9]:

$$
\begin{gathered}
\alpha=\left(\left[\sigma_{b 0}-\sigma_{c 0}\right]-1\right) /\left(\left[2 \sigma_{b 0}-2 \sigma_{c 0}\right]-1\right) ; \\
0 \leq \alpha \leq 0.5 \\
\beta=\left(\sigma_{c 0} / \sigma_{t 0}\right)(\alpha-1)-(1+\alpha) \\
\gamma=3\left(1-K_{c}\right) /\left(2 K_{c}-1\right) ; 0.5<K_{C} \leq 1.0
\end{gathered}
$$

La resistencia a la tensión y a la compresión uniaxial son dadas por $\sigma_{c 0}$ y $\sigma_{t 0}$ y pueden tomarse directamente desde datos experimentales. 


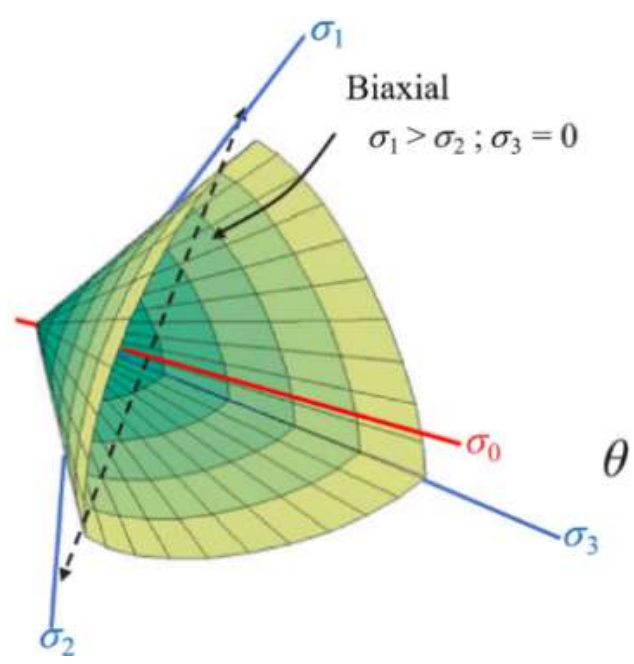

Figura 4. Superficie de cedencia Barcelona. La curvatura es ajustada al rango entre la mínima dependencia en el esfuerzo principal intermedio (e.g., Mohr-Coulomb) o la total de pendencia (e.g., Drucker Prager, un cono circular). Fuente. [10].

La resistencia de tensión biaxial $\sigma_{b 0}$ es aproximadamente $1.3 \%$ menos que la resistencia de tensión uniaxial [7] y para $\alpha$ y $\beta$ los valores típicos están entre los rangos de $0.08 \leq \alpha \leq 0.12$ y $1.10 \leq \beta \leq 1.16 \quad$ [6]. $K_{C}$ es la relación de la longitud de los meridianos de tensión y compresión para una presión dada, es decir, controla la dependencia de $\sigma_{2}$. Para $K_{C}=1, \beta$ y $\gamma$ se eliminan, dejando la función original:

$$
K_{C}=\left(\sqrt{J_{2}}\right)_{T M} /\left(\sqrt{J_{2}}\right)_{C M} \text { a una p dada }
$$

Este modelo constitutivo tiene cualidades útiles para los propósitos de las simulaciones. En primer lugar, se acomoda a un amplio rango de tipos de rocas que eran previamente modeladas por Mohr Coulomb o Drucker Prager. Segundo, en el modelado de nuevos tipos de rocas desde datos experimentales, particularmente en aplicaciones de yacimiento donde el corazonamiento es limitado, es conveniente usar falla uniaxial y datos esfuerzo-deformación para calibraciones. Tercero, el modelo es implementado en un software de elementos finitos comercial (Abaqus), el cual puede ser usado para un amplio rango de problemas geológicos [10].

\section{Pre-procesamiento}

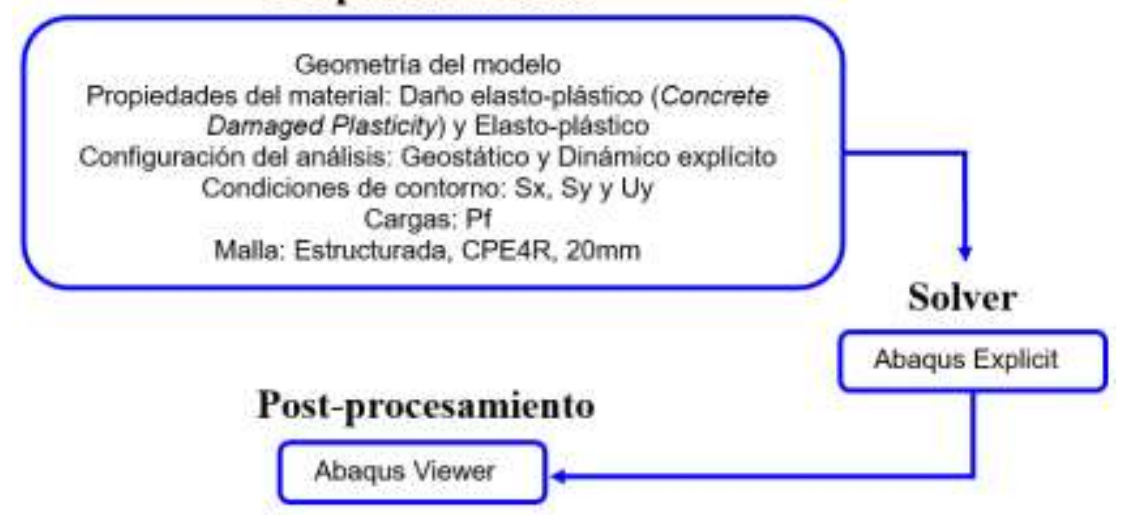

Figura 5. Procedimiento general para resolver la aplicación a fracturamiento hidráulico. Fuente. Elaboración propia.

\section{METODOLOGÍA}

Cualquier análisis usando el software comercial Abaqus involucra tres procedimientos principales: Preprocesamiento, la resolución (solver) y postprocesamiento (Figura 5), aplicable a problemas tanto bidimensionales o tridimensionales, similar al utilizado en dinámica de fluidos computacional [29], [30]. El proceso de simulación desarrollado se divide en dos fases, la primera corresponde a la propagación de la FH en un medio sin la presencia de FN y la segunda, a la propagación de una $\mathrm{FH}$ en presencia de una $\mathrm{FN}$ con el fin de evaluar su interacción y geometría final. Para las fases planteadas se tomó como base conceptual el trabajo realizado por Busetti [17], enfocado en el daño y la deformación plástica de las rocas de yacimiento. Partiendo del hecho de que las simulaciones predicen fracturas simples a través de modelos de fracturamiento hidráulico básicos y para un rango limitado de condiciones, Busetti, Mish y Reches [10] proponen que el estudio realizado puede ser aplicado a yacimientos en los cuales el proceso de fractura es mejor explicado como una red compleja o de múltiples fisuras, por lo cual es utilizado para el modelado de la interacción de FH y FN, como se desarrolla a continuación. 
Busetti, Mish y Reches [10] utilizan un enfoque para adaptar los datos de mecánica de rocas al modelo de daño de elementos finitos (Figura 6) y de esta manera generar el input del material Concrete Damaged Plasticity disponible en la librería de Abaqus. Los datos calibrados de este material se aplican al modelo de interacción FHFN planteado (Tabla 1); así como, las curvas de daño progresivo (Figura 1) y esfuerzo-deformación (Figura 7).

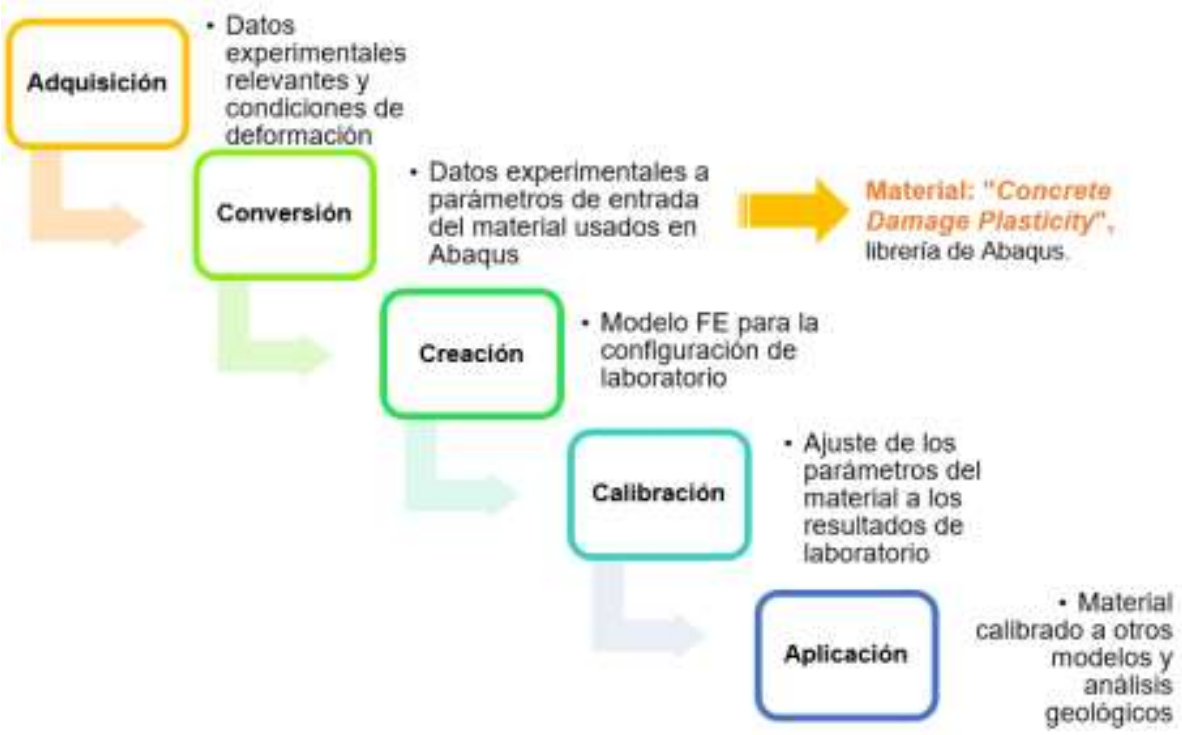

Figura 6. Enfoque para adaptar los datos de mecánica de rocas al modelo de daño de elementos finitos en Abaqus. Fuente. [10].

Utilizando algunos de los escenarios modelados para fracturamiento hidráulico de la investigación de Busetti, Mish y Reches [10] (Tabla 2) se simula la propagación de la FH con presencia de $\mathrm{FN}$ en el medio. El procedimiento general (Figura 5) para la aplicación al estudio de la interacción, incluye, una geometría con presencia de FN, cuyo espesor es $0.5 \mathrm{~mm}$ (10 veces mayor al documentado por Taleghani, González y Shojaei [18]) y fracturas naturales totalmente mineralizadas. Los modelos simulados son validados utilizando resultados experimentales publicados en la literatura.

Tabla 1. Parámetros del modelo para la reología de Berea Sandstone, SI (mm).

\begin{tabular}{|l|l|}
\hline Densidad [ton/mm $\mathbf{m}^{\mathbf{3}}$ ] & $2.1 \mathrm{E}-09$ \\
\hline Módulo de Young, E [Mpa] & 20200 \\
\hline Relación de Poisson, $\mathbf{v}$ & 0.27 \\
\hline Ángulo de dilatación, $\boldsymbol{\Psi}$ & 15 \\
\hline Excentricidad & 0.1 \\
\hline $\boldsymbol{f}_{\boldsymbol{c}_{\mathbf{0}}} / \boldsymbol{f}_{\boldsymbol{b}_{\mathbf{0}}}{ }^{*}$ & 1.16 \\
\hline Factor de intensidad de esfuerzo, $\boldsymbol{K}_{\boldsymbol{C}}$ & 0.66 \\
\hline Regularización Viscosa & 0 \\
\hline
\end{tabular}

* Relación entre el yield stress equibiaxial y uniaxial compresivo (inicial). Fuente. [17].

El modelo se enfoca en el comportamiento de la roca bajo condiciones elasto-plásticas, daño progresivo y falla, en un intento por simular realísticamente la deformación de la roca bajo condiciones de esfuerzo in-situ. Los parámetros del material del modelo numérico son derivados de las curvas de esfuerzo-deformación en tensión y comprensión de Berea Sandstone, un análogo de roca de yacimiento común [10].

Los puntos intermedios definidos en la Figura 1 y Figura 7 fueron obtenidos por Busetti [17], calibrando iterativamente el modelo de referencia contra los resultados de laboratorio, de manera que el comportamiento esfuerzo-deformación se acopla dentro de los rangos experimentales reportados para Berea Sandstone, asegurando al mismo tiempo que las definiciones para el comportamiento postyield y otros parámetros plásticos no conocidos fueran usados.

\section{CASOS DE ESTUDIO}

El modelo desarrollado es para propagación vertical de un segmento de FH dentro de una formación situada lejos de los efectos del pozo [10], cuyo fracturamiento es sensitivo al estado de esfuerzos y genera patrones de fracturamiento que van desde fracturas rectas simples hasta redes complejas. 


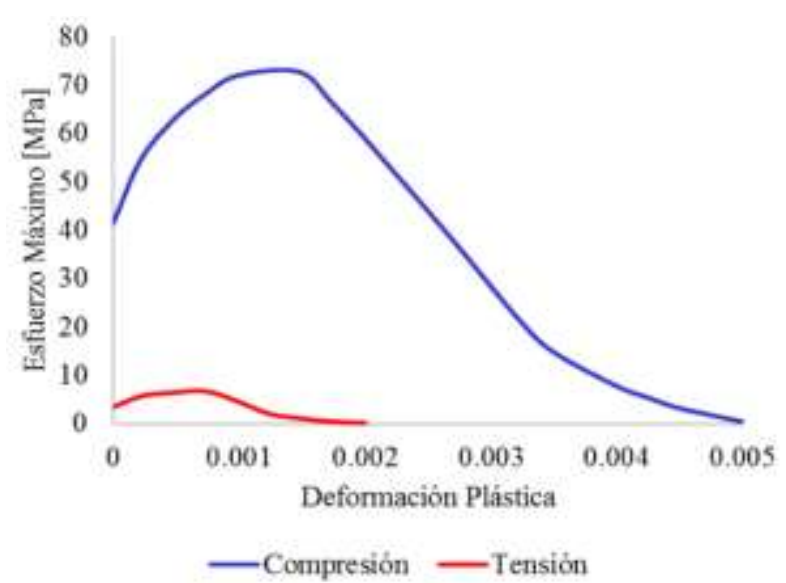

Figura 7. Curva esfuerzo-deformación post-yield. Fuente. [10].

Tradicionalmente, las fracturas por corte y tensión son definidas por el estado de esfuerzos en la superficie de la fractura comenzando con los trabajos de Coulomb en 1773 y Griffith en 1921. Por lo tanto, la aplicación de un criterio de falla relevante, por ejemplo, criterio de Mohr Coulomb, analíticamente predice la orientación de una fractura idealizada con respecto a un tensor de esfuerzos dado. Las fracturas por daño son diferentes, su inicio y propagación dependen de los campos de esfuerzo y deformación y están controladas por varios parámetros no lineales y parcialmente independientes, por ejemplo, deformación volumétrica, esfuerzos de tensión e historia de carga y deformación [10].

Tabla 2. Simulaciones de presurización interna.

\begin{tabular}{c|c|c|c|c|} 
Escenario & $\begin{array}{c}\text { Estado de } \\
\text { esfuerzos } \\
\text { tectónicos } \\
\text { (Mpa) } \\
\text { Sx - Sy }\end{array}$ & $\begin{array}{c}\text { Distribución } \\
\text { de la } \\
\text { presión } \\
\text { interna }\end{array}$ & $\begin{array}{c}\text { Patrón de la } \\
\text { trayectoria } \\
\text { principal de } \\
\text { fractura } \\
(\boldsymbol{d}>\mathbf{0 . 5})^{*}\end{array}$ & $\begin{array}{c}\text { Patrón de } \\
\text { daño } \\
\mathbf{( 0 . 0 1}\end{array}$ \\
$\boldsymbol{d}<\mathbf{0 . 1})^{* *}$ \\
\hline 1 & $10-50$ & Uniforme & Compleja, larga & $\begin{array}{c}\text { Penetrante, } \\
\text { bifurcada }\end{array}$ \\
\cline { 2 - 5 } 9 & $35-50$ & Uniforme & Larga, recta & $\begin{array}{c}\text { Pequeña } \\
\text { punta de } \\
\text { lágrima }\end{array}$ \\
\hline
\end{tabular}

* Patrón de fractura principal interpretado como los elementos que tienen un valor de daño (d) mayor de 0.5.

** Límite de daño (d) en las gráficas de contorno usadas para interpretar los patrones observados.

Fuente. [19].

El análisis de la propagación de la fractura hidráulica se realizó en un modelo de dos dimensiones (2D) con las siguientes características: La roca en estudio tiene una reología de daño elasto-plástica que se aproxima al esfuerzo, deformación finita y falla frágil observada experimentalmente en Berea Sandstone; la propagación de la fractura es determinada por el estado de daño local de la roca y, como consecuencia, la falla o fractura puede ser simulada independientemente del proceso en la punta de la fisura (la falla macroscópica obedece al proceso de fracturamiento por daño) y por último, la propagación transitoria de la fractura y la detención, ruptura, ramificación y segmentación asociadas son investigadas a través del uso de soluciones de elementos finitos dinámicas [19], [32].

La configuración del modelo es la misma utilizada en los trabajos de Busetti [17], Busetti, Mish, Hennings y Reches [19]. El modelo obedece a un plane-strain 2D de dos capas con dimensiones de $3000 \mathrm{~mm}$ (10 ft) de ancho y $2300 \mathrm{~mm}$ (7.5 ft) de alto (Figura 8). La capa inferior tiene una reología elasto-plástica de $300 \mathrm{~mm}(1 \mathrm{ft}) \mathrm{de}$ espesor y ya se encuentra fracturada por una fractura vertical (FV) de $300 \mathrm{~mm}$ de alto (1 ft) con un ancho de $0.5 \mathrm{~mm}(0.0016 \mathrm{ft})$ en la punta y $5 \mathrm{~mm}(0.016 \mathrm{ft})$ en la base del modelo.

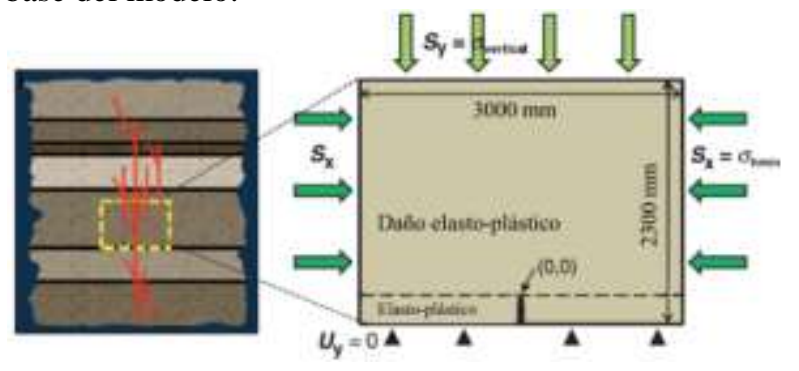

Figura 8. Escenario geológico (izquierda) y configuración del modelo de elementos finitos (derecha) para la propagación de una fractura hidráulica dentro de un estrato o capa sandstone. Fuente. [19].

La capa superior tiene una reología de daño elastoplástica que fue derivada del modelo Concrete Damaged Plasticity de Abaqus y calibrada con datos experimentales para Berea Sandstone [10], de un espesor de $2000 \mathrm{~mm}$ (6.6 ft). El modelo se carga (1) presurizando la $\mathrm{FV}$ en la capa inferior con lo cual se espera que se propague hacia arriba y (2) con esfuerzos remotos o tectónicos de régimen de falla normal $\left(\sigma_{v} \geq \sigma_{\text {hmax }} \geq\right.$ $\left.\sigma_{\text {hmin }}\right)$, el modelo representa el plano $\sigma_{v}-\sigma_{\text {hmin }}$. La superficie de cedencia plástica está establecida mediante el modelo Barcelona, el cual está basado en la plasticidad de Mohr Coulomb y usa una adaptación de DruckerPrager. Es importante mencionar que la resolución de la zona equivalente de daño para una fractura es determinada por el enmallado o distribución de la malla y que el área de localización de daño para el modelo simulado es de la dimensión de un elemento, el cual es de $20 \mathrm{~mm}(0.065 \mathrm{ft})$.

Las condiciones de contorno son los esfuerzos $S_{x}=$ $\sigma_{h \min }$ y $S_{y}=\sigma_{v}$ en los lados y en la parte superior del modelo para simular el esfuerzo horizontal mínimo y el esfuerzo vertical (sobrecarga), respectivamente, y la base está bloqueada en la dirección $y$, es decir, su desplazamiento está restringido en esa dirección. La presión dentro de la FV es aplicada para simular la inyección de fluido. En el modelo, $S_{x}, S_{y}$ y la gravedad 
(Gr) son cargadas primero, seguido por un paso de presurización, donde la presión de fractura (Pf) se incrementa hasta $2 S x$ o hasta que la solución sea inestable.

Para cada una de las simulaciones se cuantifica la cantidad de daño $d$. A menos que se especifique lo contrario, $d$ representa el daño combinado de tensión y compresión y es igual a la degradación de la rigidez local, por ejemplo, $d=0.9$ significa que el $90 \%$ de la rigidez original ha sido perdida. El parámetro $d=0$ es para roca no dañada o intacta y $d \sim 0.4$ coincide con el límite de esfuerzo final y se entiende generalmente que refleja la etapa en la que las fisuras se han unido para formar fracturas macroscópicas, y $d=1$ representa un daño penetrante o una región completamente fallada con rigidez igual a cero [19].

\subsection{Fase I: Modelado del fracturamiento hidráulico}

Se realiza a través de la aplicación de esfuerzos tectónicos, seguido por la presurización interna de la fractura vertical preexistente según las condiciones de la

Tabla 2. Para los dos escenarios seleccionados, $S_{y}$ se mantiene constante y la presión interna aumenta linealmente con el tiempo de $0 \mathrm{Mpa}$ hasta $P_{f}=2 S_{x}$, la configuración del modelo simulado es el presentado en la Figura 8. La Pf se incrementa a una tasa constante de 10 $\mathrm{Mpa} / \mathrm{s}$ (1450 psi/s), la cual es aproximadamente diez veces más rápida que la experimentada durante un bombeo típico, pero permite tiempos de solución más eficientes [19]. En los modelos simulados se toma en consideración presión uniforme en la FV. Escenarios con diferencial de esfuerzos relativamente alto (relación grande $S_{y} / S_{x}$ ) producen patrones largos de fractura hacia la capa superior (escenario 1 de la Tabla 2), así como ramificaciones y segmentos extensos debido al desarrollo de sitios de acumulación asimétricos. Los escenarios con un diferencial de esfuerzos relativamente bajo (escenario 9 de la Tabla 2) varían significativamente, en ellos se observa propagación continua y uniforme debido a los patrones de detención y ruptura consecutivos, así como a los corredores de daño simétricos. Las características morfológicas comunes en las simulaciones son presentadas en la Figura 9. Los patrones o corredores de daño reconocidos en las figuras tienen en cuenta el indicador $d$ con valores entre 0.01 y 0.1 con el fin de identificar patrones de daño penetrantes y bifurcados en la Figura 10a correspondiente a un patrón de trayectoria principal de fractura compleja y larga. Para el caso de la Figura $10 \mathrm{~b}$ es posible notar patrones de daño en forma de pequeña punta de lagrima; al igual que, un patrón de trayectoria principal de fractura larga y recta, concordando con los resultados de las investigaciones de [19].

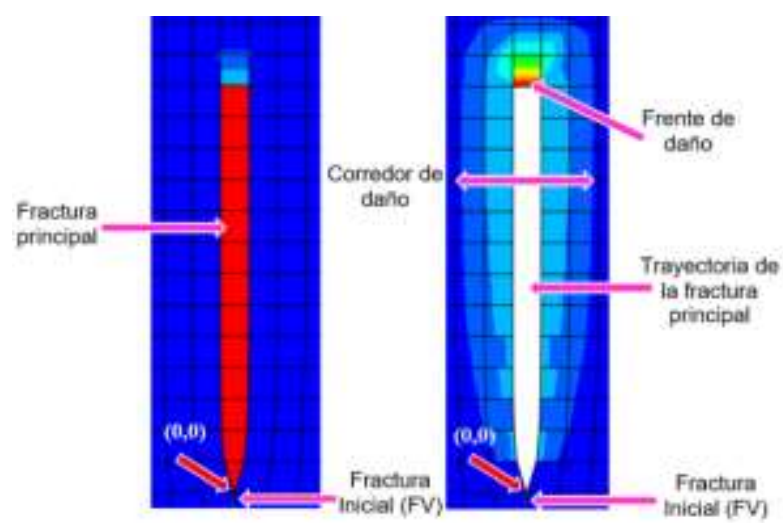

Figura 9. Características morfológicas principales. Fuente. Elaboración propia.

La intensificación de daño prolongado dentro de la propagación resulta en la formación de múltiples sitios potenciales para la ramificación y segmentación (Figura 11). Se prevé que después, cuando el fluido presurizado se propague dentro del corredor de daño (un proceso que no es simulado en esta ocasión) esos sitios de ramificación potenciales podrían llegar a ser ramas de crecimiento activo o permanecer como una pared de daño de fractura, afectando de manera considerable la geometría final de propagación y la efectividad del proceso de fracturamiento [19]. Las ramas activas crecen en longitud y podrían eventualmente vincularse a la trayectoria principal (Figura 11), generando mayor complejidad en el sistema.

Las ramas y segmentos se desarrollan a través de etapas de propagación transitoria complejas que dependen de la configuración de carga [19]. Por ejemplo, durante las simulaciones de presurización realizadas, las ramas y segmentos llegaron a ser mucho más dominantes en los estados de diferencial de esfuerzos (DS) reducido (Figura 12b). Cuando se presenta un gran diferencial de esfuerzos (Figura 12a) o un muy bajo diferencial de esfuerzos (Figura 12b) se producen redes de fracturas complejas, siendo más críticas las configuraciones resultado de altos DS. Por otro lado, cuando existe muy bajo diferencial de esfuerzos en la formación y la fractura inicia su propagación, está no encuentra un camino de propagación bien definido, por lo cual la aparición de fracturas ramificadas se hace posible (Figura 12b), lo que lleva a una disminución de su longitud y ancho, afectando el impacto positivo de la $\mathrm{FH}$ en propagación. 

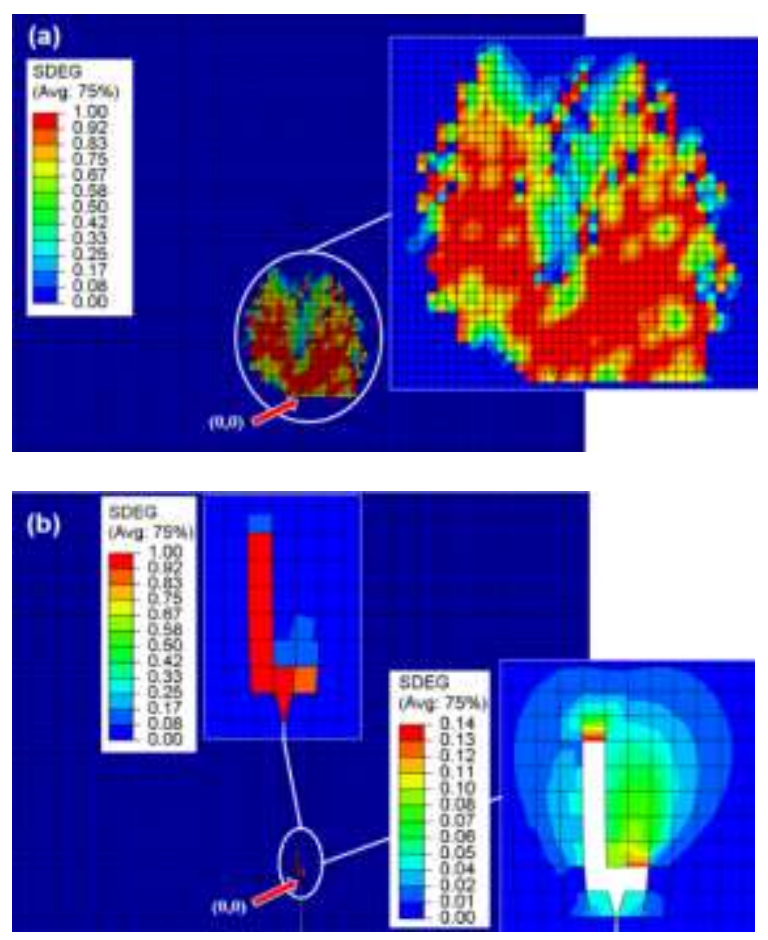

Figura 10. Presurización. Escenario $1 . S x=10 \mathrm{Mpa}, \mathrm{Pf}=2 \mathrm{Sx}$, $\mathrm{t}=1.790 \mathrm{~s}$ (a). Escenario 9. $\mathrm{Sx}=35 \mathrm{Mpa}, \mathrm{Pf}=2 \mathrm{Sx}, \mathrm{t}=7 \mathrm{~s}(\mathrm{~b})$.

Fuente. Elaboración propia.

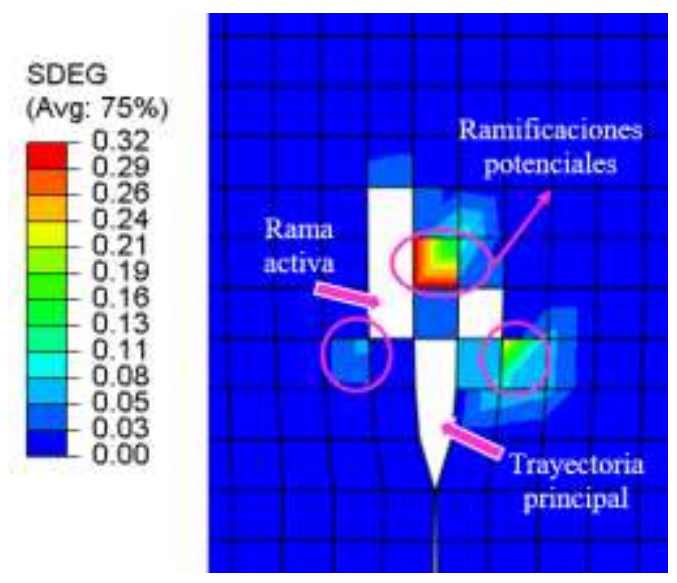

Figura 11. Presurización Escenario 1, Tabla 2. $\mathrm{Sx}=10 \mathrm{Mpa}$, $\mathrm{Pf}=2 \mathrm{Sx}, \mathrm{t}=1.780 \mathrm{~s}$. Rama activa y trayectoria principal $d \geq 0.9$ Fuente. Elaboración propia.

\subsection{Fase II: Modelado de la interacción FH-FN}

Desde la perspectiva de un yacimiento, una fractura es una discontinuidad que se encuentra en el mismo y, es producida por deformaciones mecánicas o procesos diagenéticos que ha sufrido la roca [20].

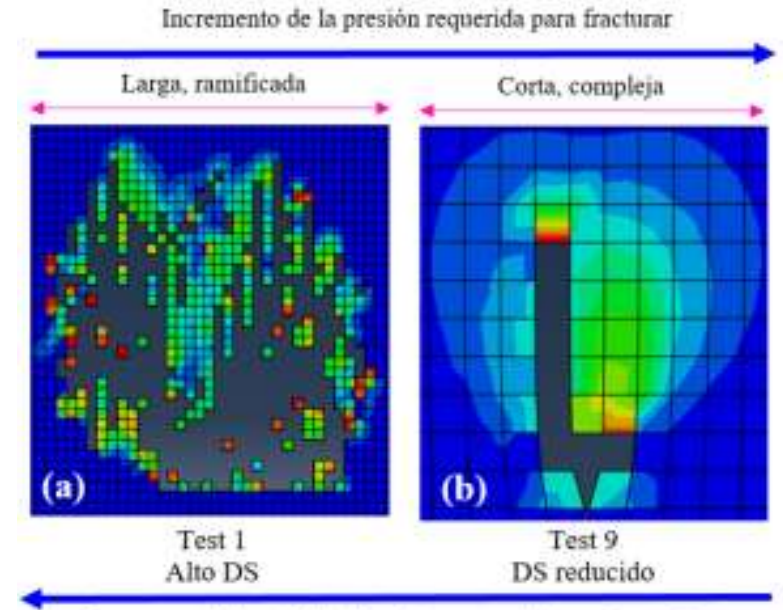

Incremento de la relación de esfuerzos tectónicos, Sy $\gg>S x$

Figura 12. Fracturas hidráulicas formadas bajo un rango de regímenes de esfuerzos. Fuente. Pruebas de presurización tomadas de la Figura 10.

La Figura 13 representa la configuración para el modelado de la interacción FH-FN y las características de ubicación espacial de cada elemento del sistema (FN, $\mathrm{FV}$, longitud y ancho, entre otras). También se utiliza la reología de daño elasto-plástica para el material-1 y elasto-plástica para el material-2 tal como en los trabajos de [10]. La configuración del modelo donde se propagará la FN es el mismo utilizado para el modelado de FH (Figura 8).

\subsection{Fase II: Fracturas mineralizadas}

Como su nombre lo indica, estas fracturas son aquellas que han sido llenadas por mineralización secundaria o diagenética; muy a menudo este material de cementación es cuarzo, carbonato o ambos y son estudiadas por ser las más comunes en yacimientos naturalmente fracturados (YNF) [20].

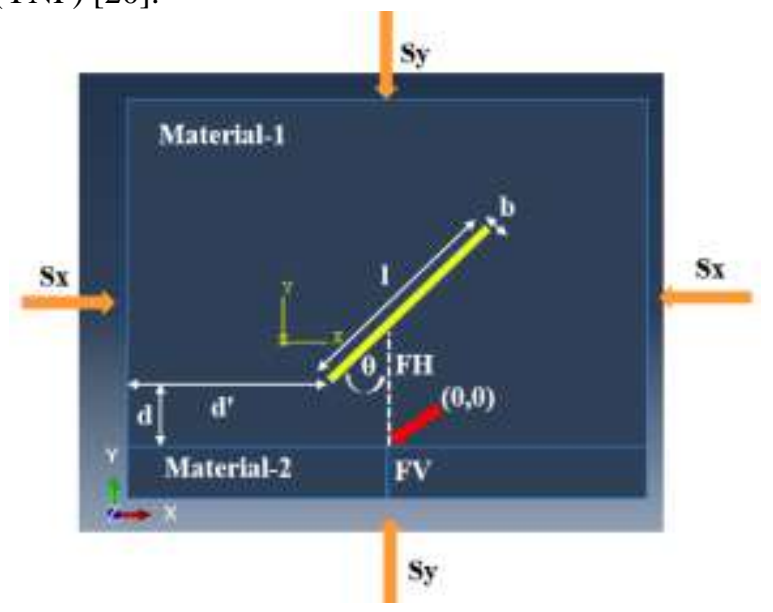

Figura 13. Modelo bidimensional de simulación. La FH se propaga hasta lograr la interacción con una FN dispuesta a un ángulo $\theta$. Fuente. Elaboración propia. 
Las simulaciones realizadas (Tabla 3) demuestran la sensibilidad del patrón de geometría de fractura al diferencial de esfuerzos y a la orientación de la FN respecto a los esfuerzos in-situ, examinando las propiedades de las $\mathrm{FN}$ totalmente mineralizadas $\mathrm{O}$ selladas que son comunes en formaciones tales como Barnett Shale, con el fin de evidenciar que sirven como una trayectoria débil para el comienzo y/o desviación de la FH. Se realizaron un total de diez simulaciones (Tabla 3) para evaluar la presencia de FN mineralizadas, cada una de ellas con un ancho de $0.5 \mathrm{~mm}$ y una longitud de $120 \mathrm{~mm}$. Para el análisis del diferencial de esfuerzos se tomaron los escenarios 1 y 9 de la (Tabla 2), para evaluar un alto y un bajo diferencial respectivamente y un par de simulaciones para un diferencial igual a cero o en estado isotrópico. La tasa de inyección para el escenario 1 es 10 $\mathrm{Mpa} / \mathrm{s}$ y $35 \mathrm{Mpa} / \mathrm{s}$ para el test 9 y el isotrópico, considerando un tiempo máximo de simulación de 2 segundos para evaluar el efecto de la Pf aplicada en la FV para cada configuración, con ángulos de interacción de $0^{\circ}, 90^{\circ}, 60^{\circ}$ y $30^{\circ}$. En cuanto al material de la FN mineralizada se considera una lutita o mudstone con una densidad de 2.0E-09 (ton $/ \mathrm{mm}^{3}$ ), un módulo de Young de 7230 (Mpa) y una relación de Poisson de 0.29 [21].

El inicio y propagación de las fracturas hidráulicas es controlado principalmente por el campo de esfuerzos insitu [22], el cual es alterado por la presencia de discontinuidades o fracturas naturales, configuración que ocasiona que la $\mathrm{FH}$ no se propague con la misma geometría o trayectoria de propagación, dependiendo de la distribución de esfuerzos en el medio y la presencia de FN, como puede observarse en la Figura 14 que compila las simulaciones realizadas según la Tabla 3 .

Tabla 3. Interacción con fracturas mineralizadas.

\begin{tabular}{|c|c|c|c|c|c|}
\hline Caso & $\begin{array}{c}\text { Estado } \\
\text { de } \sigma \\
\text { (Mpa) } \\
\text { Sx - Sy }\end{array}$ & $\begin{array}{c}\text { Tipo de } \\
\text { anisotropía }\end{array}$ & $\begin{array}{c}\text { Presión } \\
\text { de iny } \\
\text { (Mpa, } \\
\text { máx.) }\end{array}$ & $\theta$ & Descripción \\
\hline 1 & $10-50$ & Alta & 20 & 90 & $\begin{array}{l}l=120 \mathrm{~mm} \\
d=80 \mathrm{~mm} \\
t=1.730 \mathrm{~s}\end{array}$ \\
\hline 2 & $10-50$ & Alta & 20 & 0 & $\begin{array}{c}l=120 \mathrm{~mm} \\
d=80 \mathrm{~mm} \\
d^{\prime}=1540 \mathrm{~mm} \\
t=1.730 \mathrm{~s}\end{array}$ \\
\hline 3 & $10-50$ & Alta & 20 & 60 & $\begin{array}{c}l=120 \mathrm{~mm} \\
d=20 \mathrm{~mm} \\
t=1.730 \mathrm{~s}\end{array}$ \\
\hline 4 & $10-50$ & Alta & 20 & 30 & $\begin{array}{l}I=120 \mathrm{~mm} \\
d=20 \mathrm{~mm} \\
t=1.730 \mathrm{~s}\end{array}$ \\
\hline 5 & $35-50$ & Reducida & 70 & 60 & $\begin{array}{l}l=120 \mathrm{~mm} \\
d=20 \mathrm{~mm} \\
t=1.860 \mathrm{~s}\end{array}$ \\
\hline 6 & $35-50$ & Reducida & 70 & 30 & $\begin{array}{c}l=120 \mathrm{~mm} \\
d=20 \mathrm{~mm} \\
t=1.860 \mathrm{~s}\end{array}$ \\
\hline 7 & $35-35$ & Isotrópico & 70 & 90 & $\begin{array}{l}l=120 \mathrm{~mm} \\
d=80 \mathrm{~mm} \\
t=1.915 \mathrm{~s}\end{array}$ \\
\hline 8 & $35-35$ & Isotrópico & 70 & 0 & $\begin{array}{c}l=120 \mathrm{~mm} \\
d=80 \mathrm{~mm} \\
d^{\prime}=1540 \mathrm{~mm} \\
t=1.915 \mathrm{~s}\end{array}$ \\
\hline 9 & $35-35$ & Isotrópico & 70 & 60 & $\begin{array}{l}l=120 \mathrm{~mm} \\
d=20 \mathrm{~mm} \\
t=1.915 \mathrm{~s}\end{array}$ \\
\hline 10 & $35-35$ & Isotrópico & 70 & 30 & $\begin{array}{l}l=120 \mathrm{~mm} \\
d=20 \mathrm{~mm} \\
t=1.915 \mathrm{~s}\end{array}$ \\
\hline
\end{tabular}

Fuente. Elaboración propia. 
La intersección entre fracturas es menos probable si la dirección es paralela entre ellas (casos de comparación 2 y 8, Tabla 3), pero se da interacción entre cuerpos cercanos, es decir, aunque no existe contacto entre las fracturas, se altera la configuración geomecánica del medio y las FN pueden ser reactivadas por estar dentro de la zona del proceso o alrededor de la punta de la fractura, tal como la FN en el caso 2 y 8 , donde se evidencia reactivación. Analizando el caso donde la FN es ortogonal a la dirección de la FH, esta última cruza la FN sin mayor oposición (casos de comparación 1 y 7 , (Tabla 3), comportamiento similar al documentado en trabajos como el de [23] sobre la influencia de las FN en la propagación de $\mathrm{FH}$.

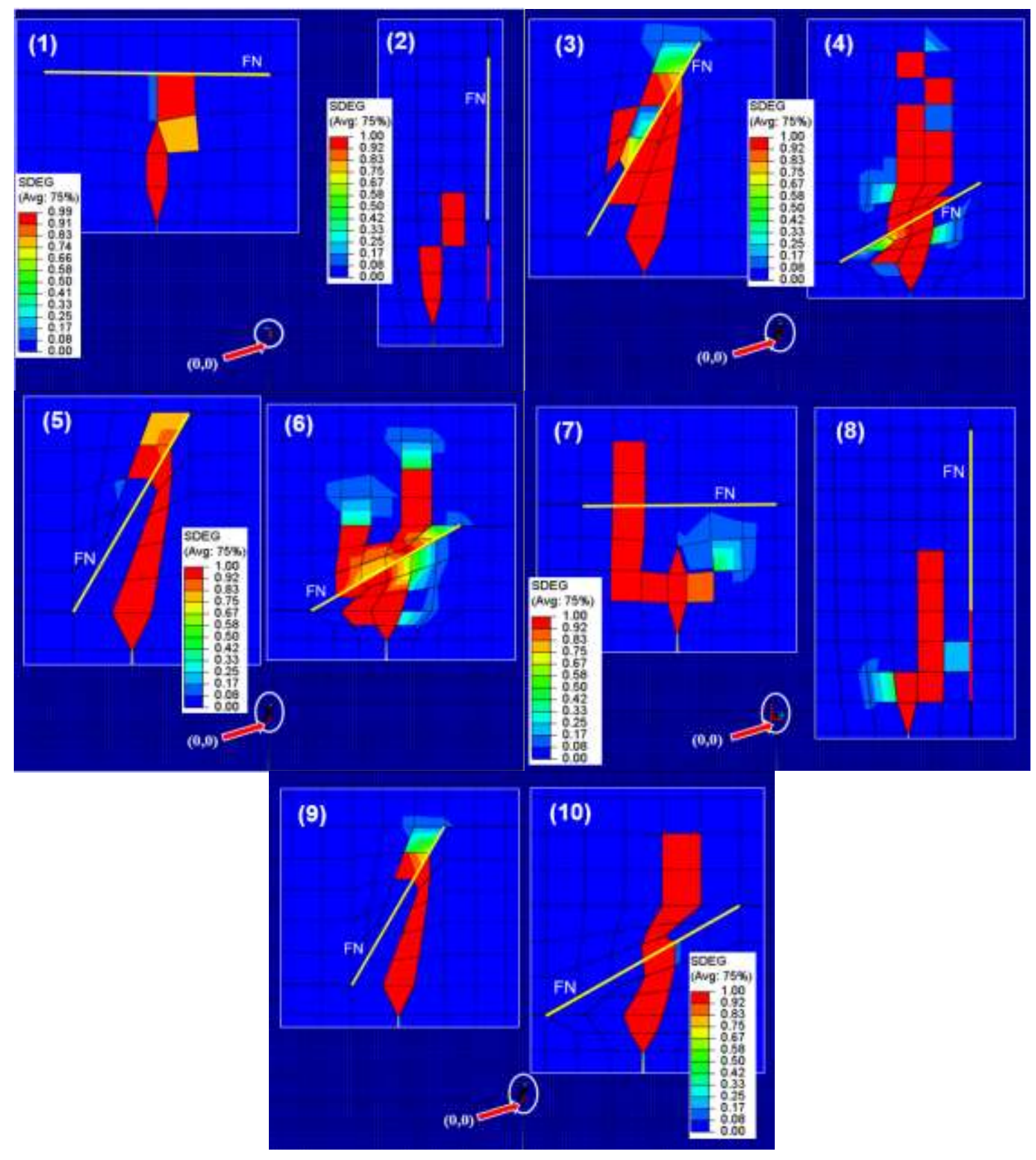

Figura 14. Modelado de la interacción FN-FH con FN mineralizadas. Casos de comparación del 1 al 10, Tabla 3. Fuente. Elaboración propia. 
Los casos de comparación de la Tabla 3, muestran que la fractura en propagación cruza la FN, se queda en ella algún tiempo (característica que puede ser observada debido al desarrollo dinámico de cada una de las simulaciones), o en algunos casos, se queda en la FN por una pequeña distancia y rompe de nuevo para propagarse en una dirección más favorable mecánicamente, dependiendo del ángulo de interacción de la FN y la FH, así como de la dirección relativa de la FN con el campo de esfuerzos; situaciones que también pueden ser evidenciadas en investigaciones experimentales como las realizadas por Blanton[25], Zhou y Xue [24].

En los trabajos realizados por Wu y Olson [26] el crecimiento de la FH es suprimido por un alto diferencial de esfuerzos, debido principalmente a la desviación de la FH a lo largo de la FN (caso de comparación 3, Tabla3). La Figura 15 contiene los datos del caso de comparación 3, 5 y 9 de la Tabla 3, la cual corresponde a una interacción con un ángulo de $60^{\circ}$ y para el cual se grafica la afectación de un diferencial de esfuerzos alto (40 Mpa), uno bajo (15 Mpa) y uno nulo, respectivamente. La Figura 15 denota que la FH que se propaga en un yacimiento con un alto diferencial de esfuerzos y en presencia de FN, tiene una penetración menor en el mismo y una apertura amplia, debido a que la propagación de la fractura se hace más lenta cuando intercepta con la FN en el caso de un alto diferencial de esfuerzos.

\subsection{Interacciones}

Las simulaciones realizadas permiten identificar diferentes escenarios de interacción entre una FH y una FN. Los escenarios de interacción más representativos incluyen una FH detenida en una FN, el cruce con o sin desplazamiento, la ramificación en la intersección con FN y la ramificación al final de una FN [27]. A través del modelado realizado en el software comercial Abaqus y haciendo uso del material Concrete Damage Plasticity y un análisis dinámico explícito; este tipo de interacciones entre fracturas es posible representarlas y evaluarlas respecto al tiempo, presentando dificultades importantes como el enmallado del medio y el gran costo computacional que se traduce en grandes tiempos de simulación.

La Figura 16 muestra la secuencia de propagación de la FH para el caso de comparación 3 de Tabla 3 , a diferentes tiempos de simulación, donde la Figura 16 d representa un mapa de la interpretación de la geometría de la FH, en el que es importante señalar que debido a que la variable daño es isotrópica, la interpretación de la dirección del crecimiento de fractura se realiza observando el enlace entre los elementos.

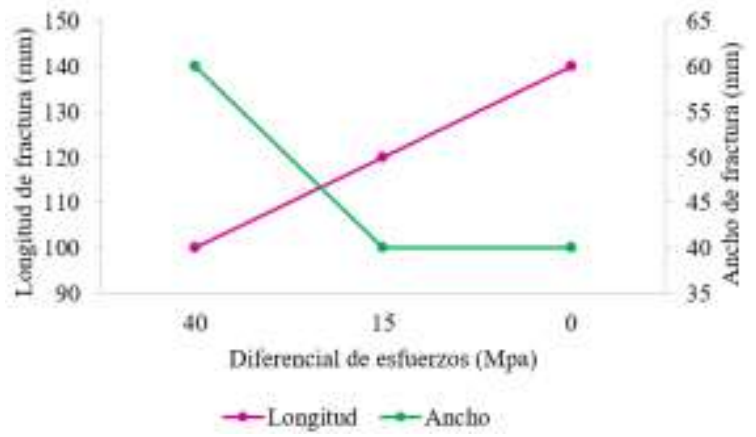

Figura 15. (a) Longitud de la fractura hidráulica y (b) ancho para tres casos de propagación de FH con diferentes diferenciales de esfuerzos en presencia de una FN con un $\theta=60^{\circ}$. Fuente. Elaboración propia.

Los números de la Figura 16d indican el orden de propagación o ramificación de la $\mathrm{FH}$, a través de ellos se hace posible la identificación de interacciones como FH detenida en una FN (\#2), cruce de FN con desplazamiento (\#3 y 4) y la ramificación en la intersección con una FN (\#5) y consecuente cruce.
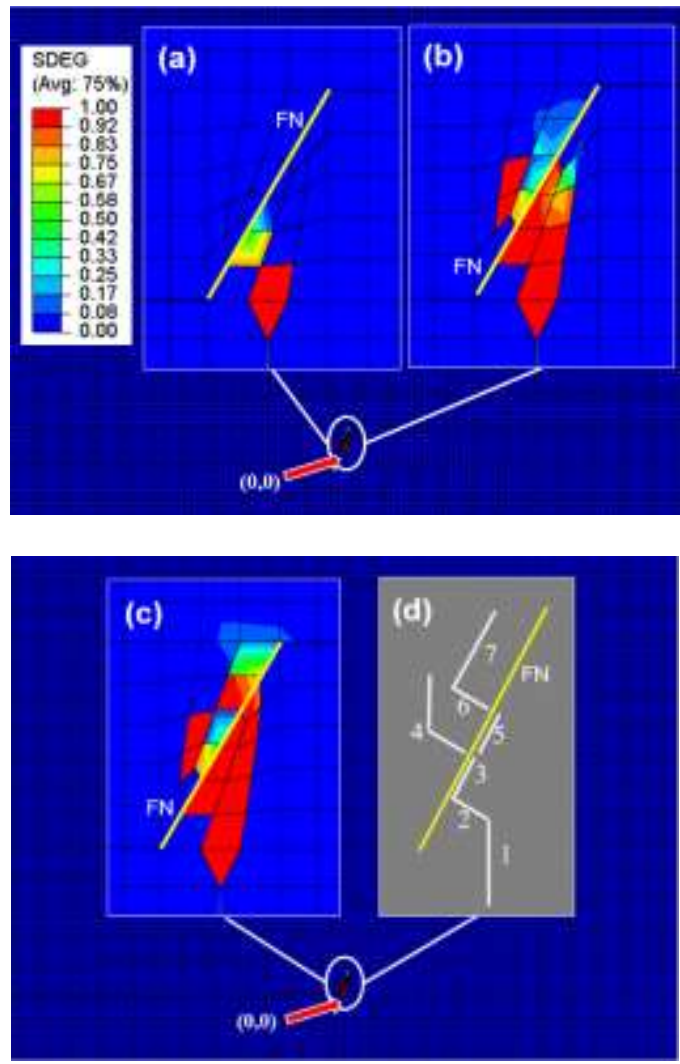

Figura 16. Secuencia de propagación de $\mathrm{FH}$ en presencia de una FN mineralizada. Caso de comparación 3 de la Tabla 3. Alto diferencial de esfuerzos y $\theta=60^{\circ}$. Fuente. Elaboración propia. 
La Figura 17 representa la secuencia de propagación para el caso de comparación 6 de la Tabla 3 donde es posible identificar a través de la Figura $17 \mathrm{~d}$ una FH detenida al encontrarse con la FN (\#2), el cruce de una FN sin desplazamiento (\#3 y 4), la ramificación en intersección con la FN (\#6 y 8) y la ramificación al final de una FN (Figura 17c).
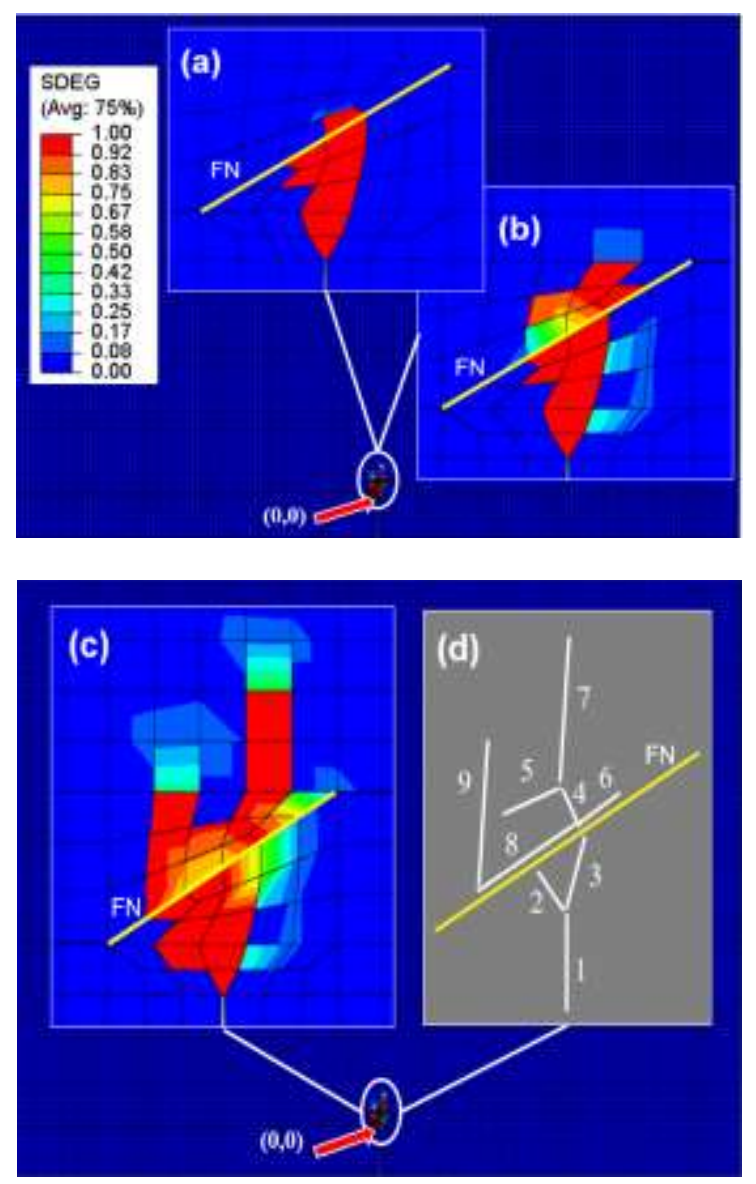

Figura 17. Secuencia de propagación de FH en presencia de una FN mineralizada. Caso de comparación 6 de la Tabla 3. Bajo diferencial de esfuerzos y $\theta=30^{\circ}$. Fuente. Elaboración propia.

Dependiendo de la configuración de los casos de estudio, cada una de las interacciones puede ser observada, teniendo en cuenta la configuración de esfuerzos, el ángulo de interacción y la Pf o presurización del medio.

Las interacciones resultantes del modelado realizado permiten sensibilizar parámetros como las condiciones de diferencial de esfuerzos y el ángulo de interacción, siendo los parámetros más influyentes en la geometría de fractura final, producto de la interacción FH-FN a través de fracturamiento hidráulico. Dicha relación FH-FN resulta en la formación de redes de fracturas complejas
(Figura 16d y Figura 17d), a través de las cuales podría mejorarse la productividad de la formación debido a la apertura de las FN y al aumento del área de contacto o volumen afectado debido al fracturamiento; en contraste con procesos de presurización que resultan poco previsibles o exitosos debido a la coalescencia de las FN en una $\mathrm{FH}$, lo que disminuye el área de afectación o volumen de contacto. Las FN son más sensitivas a esfuerzos que la matriz rocosa, debido a la configuración de los mismos a su alrededor; las FN son afectadas por disturbios de esfuerzos debido a la inyección de fluidos, resultando en apertura, cierre y reorientación de las mismas (Figura 16 y Figura 17), influyendo en las propiedades geomecánicas del medio y afectando principalmente la permeabilidad. Además, los efectos de la nueva red de fractura compleja podrían verse reflejados en pérdidas de fluido fracturante (leakoff), prematuro screen out, detención de la propagación de fractura, formación de múltiples fracturas y presiones netas altas [31]; incrementando la complejidad de los YNF y disminuyendo el éxito de la operación de fracturamiento hidráulico.

Las FN totalmente mineralizadas (simulaciones de la Tabla 3) pueden crear barreras de permeabilidad para todo tipo de flujo, lo que a su vez podría generar pequeños compartimientos dentro del yacimiento que pueden llevar a recuperaciones no económicas o marginales [33]; sin embargo, bajo diferentes condiciones (Figura 14 casos 3, 4, 6 y 7) son el inicio de una red de fracturas que lleva a un mayor volumen contactado y por ende a un aumento en la cantidad de fluido recuperado. Si las FN de la Figura 16 y Figura 17 no existieran el área afectada por dicha interacción no sería posible y el éxito del tratamiento de fracturamiento hidráulico bajo estos escenarios se reduciría, pues el objetivo principal de su aplicación en YNF es el de contactar la mayor área posible.

Daneshy [28] atribuyo el efecto de las fracturas preexistentes (FN) a su influencia en el campo de esfuerzos local, situación que claramente modifica la dirección de propagación de la FH en el medio (Figura 14). Sus experimentos al igual que el modelado realizado en esta investigación mostraron que pequeñas fracturas, abiertas o cerradas, fueron influenciadas localmente por la FH inducida, y fueron capaces de cambiar su orientación general configurando una geometría de fractura más compleja.

Los escenarios de interacción FN-FH no pueden darse de manera simultánea, pero si pueden ser observados dependiendo del valor de la presión de inyección aplicada y las características del medio, tales como diferencial de 
esfuerzos y ángulo de interacción o aproximación (e.g. Figura 16 y Figura 17).

La identificación de los escenarios de interacción mencionados por Weng [27] también es posible, analizando los resultados de las simulaciones de la Tabla 3. La Figura 14 caso 1 permite identificar bajo esas condiciones una $\mathrm{FH}$ detenida en la FN, al menos momentáneamente como resultado de un valor de presión en la FH menor que el esfuerzo normal de la FN. El cruce de una FN con o sin desplazamiento se establece en los casos 3, 4, 5, 6, 7, 9 y 10 de la Figura 14, donde para el cruce sin desplazamiento (casos 5, 7, 9 y 10) se cumple que la presión en el punto de intersección excede la presión necesaria para iniciar una fractura a lo largo de la dirección de propagación original de la FH, y para los casos con desplazamiento la presión en algún lugar de la FN es suficientemente alta para superar la resistencia de la fractura y romperse desde la FN en algún lugar entre el punto de intersección y la punta de la FN. La ramificación al final de una $\mathrm{FN}$ resultado de la interacción con una $\mathrm{FH}$ en propagación puede detallarse en los casos 3 y 6 de la Figura 14, donde la presión en uno de los extremos de la FN excede la presión neta requerida para comenzar la propagación desde la punta de la FN.

\section{CONCLUSIONES}

La implementación del modelo de daño continuo desarrollado permite identificar zonas potenciales para la ramificación y segmentación de la fractura hidráulica generada, lo que podría ocasionar efectos positivos tales como, la transformación en ramas de crecimiento activo para maximizar el alcance de la fractura hidráulica creada o efectos negativos reflejados en pérdidas de fluido fracturante o leakoff, así como un prematuro screen out, lo que hace del modelo una herramienta valiosa para la detección de este tipo de zonas críticas.

Las simulaciones capturan las principales etapas de deformación de la roca, incluyendo el comienzo de inelasticidad, strain hardening, resistencia final, strain softening y falla frágil. Debido a que la configuración del problema se establece para capturar propagación transitoria e inestable, con strain softening extremo, las simulaciones se hacen muy difíciles de ejecutar; sin embargo, es lo que convierte a la aplicación de MEF con mecánica de daño continuo en una herramienta de predicción importante y novedosa.

El efecto de las fracturas naturales en el campo de esfuerzos local es indiscutible, su existencia modifica las condiciones de propagación de la fractura hidráulica y aumenta la complejidad de la geometría final, siendo capaz de modificar la orientación original en mayor o menor medida dependiendo de las características que describan el sistema en estudio, por ejemplo, configuraciones sometidas a un gran diferencial de esfuerzos y un ángulo de interacción alto, induce el cruce de las FN con o sin desplazamiento, con menos probabilidad de alterar la trayectoria de propagación de la FH, por lo que esta cruzará un gran número de fracturas naturales a medida que se propaga a través del yacimiento, generando un sin número de geometrías y aumentando su complejidad. Las diferentes interacciones analizadas no pueden darse de manera simultánea, pero sí de manera progresiva de acuerdo con las particularidades del medio.

El nivel de penetración en el yacimiento o longitud de la geometría final alcanzada, al igual que el ancho de la misma, está influenciada por el diferencial de esfuerzos, de manera que cuando los valores del mismo son grandes, la longitud alcanzada es menor y el ancho o área influenciada es grande comparado con niveles de diferencial de esfuerzos más bajos, como resultado de una fuerte interacción con la FN y la propagación sobre ella.

\section{REFERENCIAS}

[1] J. Taheri, E. Akhgarian y A. Ghaderi, "The Effect of Hydraulic Fracture Characteristics on Production Rate in Thermal EOR Methods," Fuel, vol. 141, pp. 226-235, Feb. 2014.

[2] N. Potluri et al., "Effect of Natural Fractures on Hydraulic Fracture Propagation," SPE European Formation Damage Conference, Sheveningen, The Netherlands: Society of Petroleum Engineers, 2005, pp. 1-6.

[3] C. Cipolla et al., "Modelling Well Performance in Shale-Gas Reservoirs," SPE/EAGE Reservoir Characterization and Simulation Conference, Abu Dhabi, UAE: Society of Petroleum Engineers, 2009.

[4] M. Wangen, "Finite Element Modeling of Hydraulic Fracturing in 3D," Computational Geosciences, vol. 17, pp. 647-659, Ago. 2013.

[5] L. Li, C. Tang, G. Li, S, Wang, Z, Liang y Y. Zhang, "Numerical Simulation of 3D Hydraulic Fracturing Based on an Improved Flow-Stress-Damage Model and a Parallel FEM Technique," Rock Mechanics and Rock Engineering, vol. 45, pp. 801-818, Sep. 2012. 
[6] D. Lubliner, "A Plastic DamageModel for Concrete," International Journal of Solids and Structures, vol. 25, pp. 299-326, 1989.

[7] F. Lee y G. Fenves, "Plastic Damage Model for Cyclic Loading of Concrete Structures," Journal of Engineering Mechanics, vol. 124, pp. 892-900, Ago. 1998.

[8] Simulia, "Abaqus 6.11 Documentation. Abaqus Analysis User's Manual". [En línea]. Disponible en: http://130.149.89.49:2080/v6.11/books/usb/default.htm

[9] Simulia, "Abaqus 6.11 Documentation. Abaqus Theory Manual" [En línea]. Disponible en: http://130.149.89.49:2080/v6.11/books/stm/default.htm

[10] S. Busetti, K. Mish y Z. Reches, "Damage and Plastic Deformation of Reservoirs Rcoks: Part 1. Damage Fracturing," American Association of Petroleum Geologists, vol. 96, pp. 1687-1709, Sep. 2012.

[11] J. Jaeger y N. Cook, "Fundamentals of Rocks Mechanics”. London Chapman and Hall, pp. 593, 1976.

[12] W. Brace, B. Paulding y C. Scholz, "Dilatancy in the Fracture of Crystalline Rocks," Journal of Geophysical Research, pp. 3939-3953, Ago. 1966.

[13] S. Murrel, "The Effect of Triaxial Stress Systems on the Strength of Rocks at Atmosfheric Temperatures," Geophysical Journal International, pp. 231-281, Dic. 1965.

[14] Z. Reches y J. Dieterich, "Faulting of Rocks in Three Dimensional Strain Fields. Failure of Rocks in Polyaxial, Servo-Control Experiments," Tectonophysics, vol. 96, pp. 111-132, May. 1983.

[15] K. Mogi, "Rock Fracture," Anuual Review of Earth and Planetary Sciences, vol. 1, pp. 63-84, May. 1973.

[16] E. Alonso, A. Gens y A. Josa, "A Constitutive Model for Partially Saturated Soils," Geotechnique, vol. 40, pp. 405-430, 1990.

[17] S. Busetti, "Fracturing of Layered Reservoir Rocks," Ph.D. dissertation, University of Oklahoma, 2009.

[18] A. Taleghani, M. Gonzalez y A. Shojaei, “Overview of Numerical Models for Interactions between Hydraulic Fractures and Natural Fractures: Challenges and Limitations," Computers and Geotechnics, pp. 361-368, 2016.
[19] S. Busetti, K. Mish, P. Hennings y Z. Reches, "Damage and Plastic Deformation of Reservoir Rocks: Part 2. Propagation of a Hydraulic Fracture," American Association of Petroleum Geologists, vol. 96, pp. 17111732, Sep. 2012.

[20] R. Nelson, Geologic Analysis of Naturally Fractured Reservoirs, 2 ed. Houston, TX.: Gulf Prefessional Publishing, 2001.

[21] G. Shen, X. Shen y S. Wang, "Numerical and Experimental Studies on Fracture Propagation at a Bimaterial Interface and Its Application to Hydraulic Fracturing," American Rock Mechanics Association, Jun. 2014.

[22] D. Healy, "Hydraulic Fracturing or "Fracking": A Short Summary of Current Knowledge and Potential Environmental Impacts," Science, Technology, Research \& Innovation for the Environment Programme, Jul. 2012.

[23] J. Olson y A. Dahi-Taleghani, "The Influence of Natural Fractures on Hydraulic Fracture Propagation," AAPG, Datapages, Inc. [En línea]. Disponible en: http://www.searchanddiscovery.com/pdfz/documents/20 10/40583olson/ndx_olson.pdf.html

[24] J. Zhou y C. Xue, "Experimental Investigation of Fracture Interaction between Natural Fractures and Hydraulic Fracture in Naturally Fractured Reservoirs," SPE EUROPEC/EAGE Annual Conference and Exhibition, Vienna, Austria: Society of Petroleum Engineers, 2011.

[25] T. Blanton, “An Experimental Study of Interaction Between Hydraulically Induced and Pre-Existing Fractures," SPE Unconventional Gas Recovery Symposium, Pittsburgh, Pennsylvania: Society of Petroleum Engineers, 1982.

[26] K. Wu y J. Olson, "Numerical Investigation of Complex Hydraulic-Fracture Development in Naturally Fractured Reservoirs," SPE Hydraulic Fracturing Technology Conference, The Woodlands, Texas, USA, 2016.

[27] X. Weng, "Modeling of Complex Hydraulic Fractures in Naturally Fractured Formation," Journal of Unconventional Oil and Gas Resources, vol. 9, pp. 114135, Mar. 2015.

[28] A. Daneshy, "Hydraulic Fracture Propagation in the Presence of Planes of Weakness," SPE European Spring Meeting, Amsterdam, Netherlands: Society of Petroleum Engineers, 1974. 
[29] G. González, E. Matos, W. Martignoni y M. Mori. "The Importance of 3D Mesh Generation for Large Eddy Simulation of Gas-Solid Turbulent Flows in a Fluidized Beds," World Academy of Science, Engineering and Technology International Journal of Chemical and Molecular Engineering, vol. 6, pp. 770-777, 2012.

[30] G. González, N. Prieto y O. Salazar, "Fluid Dynamics of Gas - Solid Fluidized Beds," en Advanced Fluid Dynamics, 1a ed. Intech, 2012, cap. 3, pp. 39-58.

[31] F. Mavares y A. Pertuz, "Cementación de Revestidor en Flujo de Gas/Agua Presurizado Superficial no Esperado en un Campo de Desarrollo Costa Afuera: Rio de Janeiro, Brasil," Rev. UIS Ing., vol. 16, no. 2, pp. 79-92, 2017.

[32] W. Rodríguez, R. Rojas, José Yépez y M, Pallares, "Ánalisis de Sensibilidad y de Estabilidad Numérica en el Cálculo de Factores de Intensidad de Tensiones en un Caso de Mecánica de Fractura," Rev. UIS Ing, vol. 16, no. 2, pp. 151-160, 2017.

[33] D. González, C. Villabona, H. Vargas, E. Ariza, C. Roa y C. Barajas, "Métodos para el Control e Inhibición de la Acumulación de Depósitos Parafínicos," Rev. UIS Ing, vol. 9, no. 2, pp. 193-206, 2010. 